\title{
An Overview of the Treatment of Symptomatic Common Femoral Artery Lesions with a Focus on Endovascular Therapy
}

This article was published in the following Dove Press journal: Vascular Health and Risk Management

\section{Nicolas W Shammas (ID \\ Amanda Abi Doumet \\ Rusina Karia \\ Rommy Khalafallah}

Midwest Cardiovascular Research Foundation, Davenport, IA, USA
Correspondence: Nicolas W Shammas Midwest Cardiovascular Research Foundation, I622 E Lombard Street, Davenport, IA 52803, USA

Emailshammas@mchsi.com

\begin{abstract}
Endarterectomy of common femoral artery lesions (CFA) carries favorable longterm results and is currently still considered the gold standard for treating these lesions. Although routine stenting has been considered an option for treating the CFA, it has yielded conflicting results and is currently reserved for a bailout of suboptimal endovascular results. Newer therapies with atherectomy or lithoplasty in conjunction with pharmacologic antiproliferative therapies are promising with less bailout stenting and dissections but randomized trials are needed to confirm their effectiveness and safety.
\end{abstract}

Keywords: common femoral artery, angioplasty, stent, endarterectomy, atherectomy

\section{Introduction}

Surgery has been considered the standard treatment for symptomatic common femoral artery (CFA) disease. Endovascular therapies, however, have significantly evolved in the past recent years. Several new tools have been introduced including atherectomy devices, shockwave Lithoplasty (Shockwave Medical, Santa Clara, CA) and scoring or cutting balloons that have the potential to improve the acute procedural success and reduce dissections and bail out stenting when treating complex lesions. Furthermore, drug-coated balloons have significantly improved the long-term durability of the procedure and have become an effective therapy against restenosis. In this review, we focused on studies published within the past 10 years which included endovascular or surgical treatment of the CFA. To be eligible for inclusion, only prospective studies or retrospective studies of prospectively collected data and with a minimum of 10 treated CFAs were considered. A summary of published data is in Table 1 .

\section{Endarterectomy}

Ballota et $\mathrm{al}^{1}$ reported on a series of patients with isolated CFA disease. In this prospective series, 117 patients were enrolled. Isolated CFA endarterectomy was performed in 38 patients (31\%). Sixty percent of all patients presented with claudication and $40 \%$ with CLI. Technical success was achieved in $100 \%$ of patients. During the peri-operative (30-day) period, no death or major complications occurred. There were $8(6.6 \%)$ minor complications (self-resolving inguinal lymph leaks). Ankle brachial index (ABI) improved significantly (from 0.57 to 0.94 in claudicants and 0.42 to 0.75 in critical limb ischemia (CLI) patients). At a mean 


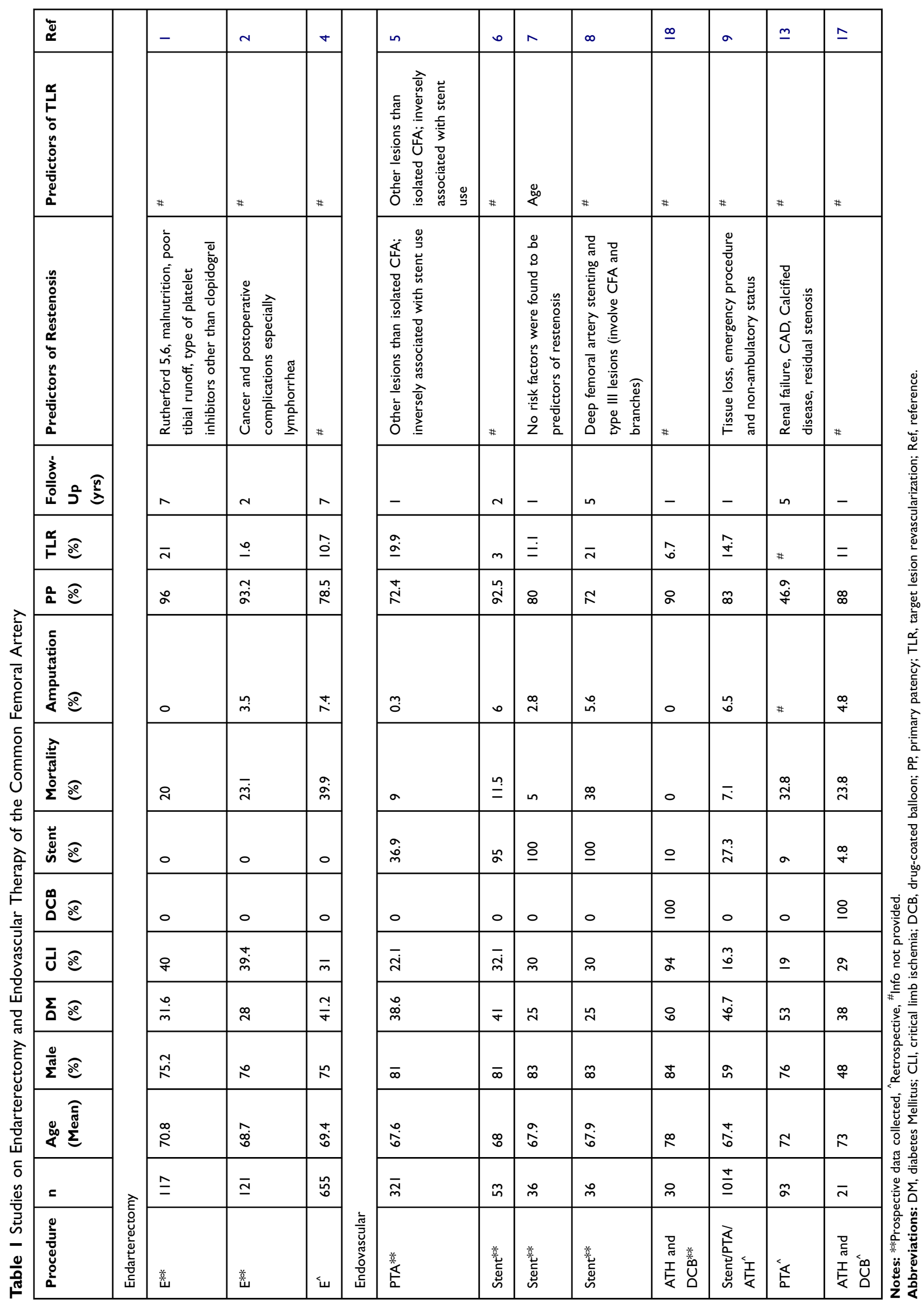


of 4.2 years, primary patency rates were $100 \%$ at 1 year and $96 \%$ at 7 years. There were 2 primary failures $(>70 \%$ CFA restenosis) that occurred at 33 and 47 months after the procedure and were treated by balloon angioplasty (PTA) without stenting. No patients needed major amputation during the follow-up. The survival rates were $100 \%$ at 1 year and $80 \%$ at 7 years. Long-term predictors of failure were Rutherford 5 and 6, type of platelet aggregation inhibitors other than clopidogrel, malnutrition, and poor tibial runoff.

Dufranc et $\mathrm{al}^{2}$ explored the endarterectomy of the CFA using the eversion technique in 121 patients enrolled prospectively. Of these 121 patients, 10 patients had isolated CFA lesions. $60.6 \%$ of patients presented with claudication and $39.4 \%$ presented with critical limb ischemia. Femoral endarterectomy was performed on 147 limbs with a technical success rate of $93.2 \%$.

Dufranc et al noted $12(8.2 \%)$ overall complications, of which $4(2.8 \%)$ were vascular and $6(4 \%)$ were local. The in-hospital mortality at 30 days was $0 \%$. At 1 year, six cases developed femoral restenosis, three involving the CFA. At 2 years, the overall survival rate was $76.9 \%$. Clinical improvement was noted in $77.9 \%$ and primary patency was $93.2 \%$. Limb salvage was $96.5 \% ; 100 \%$ in claudicants and $88.6 \%$ in the CLI patients. Identified risk factors for major amputation included malnutrition, preoperative platelet count $>45010^{9} / \mathrm{L}$, poor tibial runoff, and platelet aggregation inhibitor treatment other than clopidogrel. Protective factors associated with decreased need for secondary interventions were good tibial runoff (more than two-vessel tibial runoff), statin therapy, and extensive endarterectomy involving the CFA, superficial femoral artery (SFA), and profunda femoris artery (PFA).

In order to define the predictors of postoperative complications from endarterectomy, Nguyen et $\mathrm{al}^{3}{ }^{3}$ in a retrospective analysis, evaluated the 30-day outcomes in 1843 CFA endarterectomy patients from the National Surgical Quality Improvement Program database between 2005 and 2010. The average operative time was 146 mins with $10 \%$ of patients had to return to the operating room. The mean length of hospital stay was 4 days. Postoperative mortality and wound-related complications were $3.4 \%$ and $8 \%$ with $30 \%$ and $86 \%$ of which occurred following hospital discharge, respectively. The independent predictors of 30-day mortality were age, poor functional status, endstage renal disease, sepsis, emergency nature of the procedure, and American Society of Anesthesiologists Physical Status Classification 4 or 5 . The authors concluded that
CFA endarterectomy is not a benign procedure and a careful selection of patient is important particularly patients with multiple comorbidities.

The hybrid approach has been used by some operators depending on lesion complexity.

In a retrospective study, Wieker et $\mathrm{al}^{4}$ investigated the long-term outcome of CFA endarterectomy in 655 patients (713 vessels). Critical limb ischemia was present in 221 patients. A hybrid approach was used in 255 limbs $(35.8 \%)$. Primary patency was $78.5 \%$ and secondary patency was $89.1 \%$ at 7 years. Patency rates were $97.3 \%$ and $90.2 \%$ at 6 months and 3 years, respectively. Patency rates at 7 years were similar for a non-hybrid or hybrid approach $(78.1 \%$ vs $78.6 \% ; \mathrm{P}=0.22)$ and for critical limb ischemia vs claudication $(76.3 \%$ vs $79.4 \% ; \mathrm{P}=0.20)$. Freedom from amputation was $92.6 \%$ and procedurerelated complications were $11.5 \%$ during 7 years of follow-up. The hybrid approach has not been evaluated in a prospective study.

In summary, prospective registries show a very high success rate $(93.2 \%$ to $100 \%)$ in treating the CFA with endarterectomy. Also, a low rate of complications was seen in these studies although traditionally CFA endarterectomy is associated with wound infections, hematomas, or seromas affecting $>15 \%$ of patients. ${ }^{1}$ Finally, sustained primary patency ( $93 \%$ to $96 \%)$ and low TLR rates $(9 \%$ to $18.3 \%$ ) were seen with long term follow-up. These results are highly favorable and set a high standard for the treatment of the CFA. It should be noted, however, that these studies are limited by being single center and likely with selection bias performed by experienced operators.

\section{Endovascular Therapies of the CFA}

The endovascular treatment of CFA disease has recently gained significant momentum. The endovascular approach has been criticized as suboptimal when compared to surgery. Bonvini et $\mathrm{al}^{5}$ published a retrospective analysis of prospectively maintained single-center database to study the success of endovascular treatment of CFA Lesions. A total of 321 patients were included in this study, 140 (38.9\%) of which were true CFA bifurcation lesions. Patients with chronic total occlusion (CTO) were $17.8 \%$ and those with associated chronic SFA occlusion were $21.9 \%$. Out of 360 procedures performed, 98.6\% were with PTA (mean balloon diameter $6.55 \pm$ $0.8 \mathrm{~mm}$ ) making it the primary method of intervention. Stenting was performed in $37 \%$ of lesions as a bailout procedure when $>50 \%$ residual stenosis was noted. 
Atherectomy and kissing balloon angioplasty made up $8.3 \%$ of the interventions. Procedural success defined as $<30 \%$ angiographic evidence of residual stenosis was 93\%. Follow-up was done at 1 year (10.3 \pm 5.4 months $)$. Restenosis ( $>50 \%$ narrowing) and TLR rates were $27.6 \%$ and $19.9 \%$, respectively. When intervention involved the CFA and another infrainguinal procedure in the same setting, the 1-year TLR (OR: 1.97; 95\% CI: 1.12 to $3.44 ; \mathrm{p}=0.015)$ and procedural failure (OR: $2.71 ; 95 \%$ CI: 1.19 to $6.15 ; \mathrm{p}=0.013$ ) were higher compared to treating only the CFA bifurcation. The rates of major amputations in hospital, at 1 year, and at $>18$ months were $0 \%, 1 \%$ and $2 \%$, respectively. Finally, the rate of in-hospital death, 1-year death (8.2 \pm 5.6 months), and late death ( $>18$ months) were $4 \%, 9 \%$ and $9 \%$, respectively. This study illustrates a higher rate of TLR and lower patency rates when compared to historic numbers from endarterectomy. The study, however, was performed predominantly with angioplasty and no drugcoated balloons (DCB) or routine stenting used. Also given that no vessel prepping with atherectomy or specialty balloons was performed, a high rate of bailout stenting was noted.

Thiney et $\mathrm{al}^{6}$ published a single-center, nonrandomized, prospective study of 53 patients with the objective to report midterm outcomes of stenting of the CFA. Lesions were classified according to Azema et al ${ }^{7}$ into four groups: I $(8,15 \%)$ - disease to the CFA extended to external iliac lesions; II $(19,35.8 \%)$ - isolated lesions of the CFA; III $(22,41.5 \%)$ - lesions of the femoral bifurcation; IV $(4,7.5 \%)$ - proximal or distal anastomotic lesions bypass surgery. Out of 53 procedures performed, 50 (95\%) were done with stenting. Several types of stents were used including self-expandable stent $33(66 \%)$ and balloonexpandable stents $23(46 \%)$. Three $(5 \%)$ patients were treated with angioplasty only. The technical success rate defined as $<30 \%$ residual stenosis was $98 \%$ and the one case of failure was a type III lesion with CTO. Follow-up was done at a mean of 24 months when restenosis rate (defined by duplex ultrasound as a peak systolic velocity index $>2.4$ at the target lesion) was $7.5 \%$ and TLR rate was $4 \%$. The low rates of restenosis and TLR were likely due to stenting as the primary intervention. Sixty-seven percent of the entire cohort showed clinical improvement and $6 \%$ rate of major amputation was noted. There were four cases $(9 \%)$ of stent fracture reported out of which three were nitinol stents and one was balloon-expandable stent. These results were encouraging but could also reflect selection bias and a higher level of expertise by the operators.

Azema et $\mathrm{al}^{7}$ and Nasr et $\mathrm{al}^{8}$ published a prospective study on 36 patients (40 limbs) treated with stenting in the CFA. An early report was published by Azema et al $^{7}$ with a follow-up at a mean of 22 months followed by Nasr et $\mathrm{al}^{8}$ at a mean follow-up of 64 months. CFA lesions were classified into type I: $20 \%$ - disease to the CFA extended to external iliac lesions; type II: $42.5 \%$ - isolated lesions of the CFA; type III: $25 \%$ - lesions of the femoral bifurcation; type IV: $12.5 \%$ - proximal or distal anastomotic lesions bypass surgery. All lesions were stented with balloon-expandable 5 (11.6\%) or self-expandable $38(88.3 \%)$ stents. Primary patency, defined as patency without any percutaneous or surgical intervention in the CFA or in the adjacent areas, was $76 \%$ and $72 \%$ at 3 years and 5 years, respectively. Primary sustained clinical improvement defined as a sustained upward shift of 1 category of the Rutherford classification without the need for repeated TLR in surviving patients was $80 \%, 77 \%$ and $73 \%$ at 1 year, 3 years and 5 years, respectively. Secondary sustained clinical improvement defined as a sustained upward shift of 1 category of the Rutherford classification including the need for repeated TLR in surviving patients was $90 \%, 82 \%$ and $80 \%$ at 1 year, 3 years and 5 years, respectively.

Survival rates at 3 and 5 years were $74 \%$ and $62 \%$ unrelated to the CFA intervention. Restenosis rate (restenosis $>50 \%$ and by a peak systolic velocity index $>2.4$ at the target lesion assessed by duplex ultrasound) was $28 \%$ (11 cases) out of which $72 \%$ (8 cases) occurred at 1 year and $27 \%$ ( 3 cases) occurred after $>2$ years. There were 2 risk factors associated with restenosis; type III lesions $(\mathrm{P}=0.014 ; 95 \% \mathrm{CI}=1.53-36.73)$ and stenting of the PFA $(\mathrm{P}=0.0007 ; \mathrm{CI}=3.29-342.5)$. The TLR rates at 1 year, 3 years and 5 years were $15 \%, 17 \%$ and $21 \%$. Two major amputations were reported at 30 days and 39 months. At 6 months, one stent fracture was noted in a type I lesion treated by nitinol self-expanding stent. There was in-stent restenosis of this fracture at 12 months. The overall patency and TLR rates in the Azema ${ }^{7}$ and Nasr ${ }^{8}$ studies were not as encouraging as reported by Thiney. ${ }^{6}$ This could be related to significant differences between the cohorts of patients enrolled as well as stent types and procedural methods.

Data from the Vascular Quality Initiative (2010-2015, $\left.{ }^{9}\right)$, a multicenter prospective database, were analyzed for endovascular interventions of the CFA and PFA. A total of 1014 
patients were included. Isolated CFA intervention was noted in 946 (93.3\%) patients.

Claudication was present in $67 \%$ of patients. Access site hematoma was reported in $5.2 \%$ of patients, whereas distal embolization and perforations were seen in $0.7 \%$ and $0.6 \%$, respectively. At 1 - and 3-year follow-up survival was $92.9 \%$ and $87.2 \%$, respectively. At 1-year amputation-free survival, freedom from loss of patency or death, and reintervention-free survival were $93.5 \%, 83 \%$, and $87.5 \%$, respectively. In this analysis, the authors concluded that compared to historic controls of endarterectomy, endovascular interventions of the CFA appear to have a higher rate of reintervention and lower patency rates on a 1-year follow-up.

Linni et $\mathrm{al}^{10}$ tested bioresorbable stents versus endarterectomy in a single-center, open-label randomized trial. Of 116 patients recruited, 80 patients were enrolled (52 men, mean age $72.2 \pm 9.6$ years) and were randomized $1: 1$ to the stent versus surgery. Fifty percent of patients were smokers and $30 \%$ diabetics. The primary endpoint was surgical site infections. Some of the secondary outcome measures included patency, limb salvage and survival. CFA lesions were classified as either isolated CFA lesions, combined CFA/proximal SFA lesions, combined CFA/PFA lesions, or combined CFA/PFA/proximal SFA lesions. Isolated CFA lesions, were present in 31 patients. Both groups were well matched to demographics, cardiovascular risk factors and the presence of CFA occlusions. Infections occurred in 7 surgical patients (minor) and none in the stent group ( $\mathrm{p}<0.001)$. Technical success was $97.5 \%$ with surgery and $100 \%$ with stenting. Patency at 1 year was $80 \%$ for the stent group versus $100 \%$ for endarterectomy $(\mathrm{p}=0.007)$. There were no differences in amputation and mortality rates at 1 year.

Goueffic et $\mathrm{al}^{11}$ reported the findings of the TECCO trial; a multicenter, prospective trial that included 117 patients with de novo CFA disease randomized to endarterectomy $(n=61)$ versus stenting $(n=56)$. Thirty-four patients had isolated CFA lesions. Forty-six percent of the study population were smokers. Diabetes was present in $41 \%$ of the surgery group and $31 \%$ of the stent group. The primary outcome was the morbidity and mortality rate within 30 days. Morbidity was defined as complications that required re-intervention or prolonged hospitalization, lymphorrhea of more than 3 days, and post-operative paresthesia requiring pharmacologic intervention. The median follow-up was 2 years. The primary outcome events occurred in $26 \%$ of the endarterectomy group and
$12.5 \%$ of the stent group $(\mathrm{p}=0.05)$. The stent group had a shorter hospital stay $(3.2 \pm$ days vs $6.3 \pm 3$ days; $\mathrm{p}<$ $0.0001)$. At 2 years there were no differences in the primary patency (HR 1.7; 95\% CI, 0.5-5.6, $\mathrm{p}=0.42$ ) or TLR (HR 0.9; 95\% CI, 0.3-2.5, $\mathrm{p}=0.83$ ) rates between the 2 groups. The TECCO trial demonstrated that endovascular therapy with stenting is a viable alternative treatment to endarterectomy.

A recent metaanalysis ${ }^{12}$ evaluating outcomes of endovascular therapy versus endarterectomy of the CFA showed that the TLR rate at 1 year was $8 \%(95 \%$ CI 4-13\%) for routine stenting, $19 \%$ (95\% CI $14-23 \%)$ for selective stenting, and 4.5\% (95\% CI 1-9\%) endarterectomy. Also at 1 year, no statistical difference in primary patency was seen in the routine stent group versus the surgical group. Complication rates were, however, significantly higher with surgery and a higher mortality was seen with endarterectomy at maximum follow-up when compared to routine stenting $(23.1 \%$ (95\% CI $14-33 \%)$ versus $5.3 \%$ (95\% CI $1-11 \%)$ respectively). This analysis included both retrospective and prospective studies and selection bias cannot be excluded. However, it supports endovascular therapy as a viable option for treating the CFA as seen in the TECCO trial.

In a large retrospective analysis of 1014 patients by Siracuse et al, ${ }^{9}$ periprocedural complications of endovascular therapy were mostly vascular-related complications including hematoma, arterial dissection or stenosis/occlusion, distal embolization and perforation. Thirty-day mortality was $1.6 \%$. Predictors of mortality were advanced lung disease, tissue loss, dialysis, emergency procedure, and older age. Aspirin was protective. In addition, predictors of amputation were advanced lung disease, tissue loss, dialysis, stent use, nonambulatory status, and female sex. Also, in a small retrospective study, Soga et al ${ }^{13}$ noted that predictors of patency loss with endovascular therapy were renal failure, coronary artery disease, calcified disease, and a higher residual stenosis.

The data above indicate that balloon angioplasty to the CFA with bailout stenting is not a favorable strategy when compared to endarterectomy. Data on routine stenting provide conflicting results but overall it appears that patency and TLR rates are improved when compared to bailout stenting on short-term follow up (1-2 years). Numerically, however, the rates of TLR and restenosis continue to favor surgery and long-term data for stenting is still lacking. Although routine stenting may yield favorable outcomes, the use of stent in the CFA is not generally preferred as a primary strategy because 


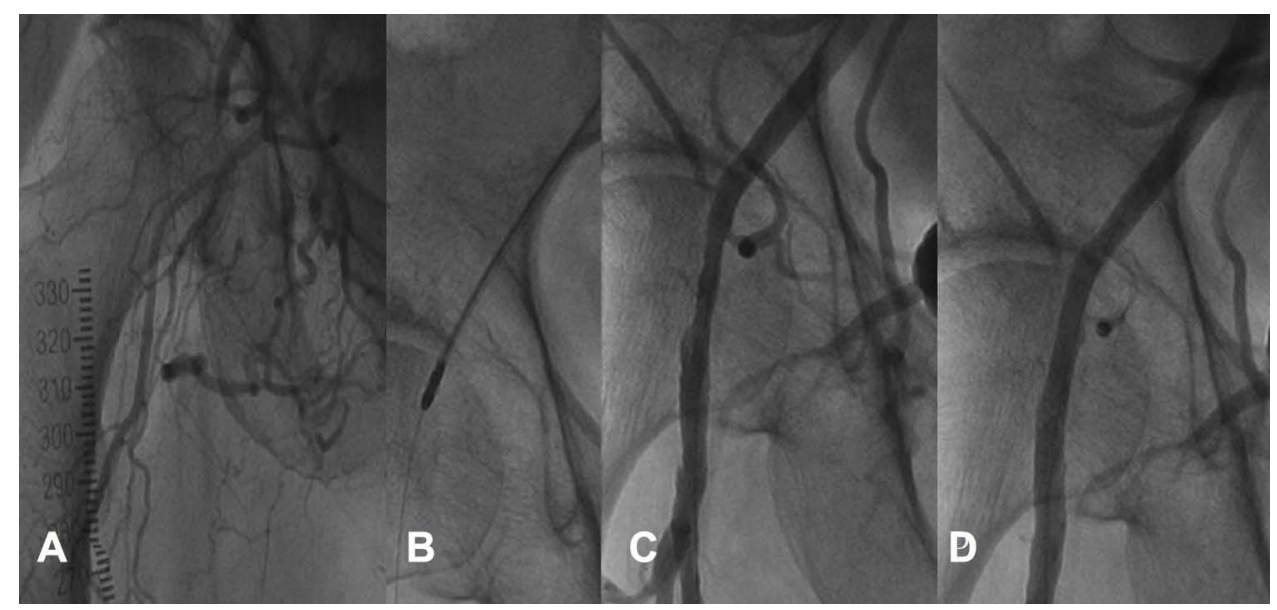

Figure I (A). Chronic total occlusion of the right common femoral artery. (B). Jetstream atherectomy (Boston Scientific, Maples Grove, MN, IL). (C). Post Jetstream atherectomy with no adjunctive therapy. (D). Post Drug-coated balloon following Jetstream atherectomy.

of stent fractures and the prevention of future targets for bypass surgery. Also, the presence of a CFA stent may become a relative problem for future access.

\section{Future Directions}

The advent of new endovascular technologies appears to be promising for treating the CFA. Debulking with atherectomy (Figure 1) remains promising particularly in calcified vessels with severe and eccentric lesions. Less bailout stenting is expected and some early data suggest improved patency and freedom from TLR when compared to angioplasty and bailout stenting. ${ }^{14,15}$ However, there are conflicting data on the use of atherectomy with adjunctive DCB in the CFA. In a two-center retrospective study, ${ }^{16}$ 154 patients with CFA disease were treated with DCB $(n=47)$ or balloon angioplasty and bailout stenting $(n=107)$. Critical limb ischemia and moderate to severe lesion calcification were present in $43 \%$ and $75 \%$ of patients, respectively. A high use of vessel prepping with atherectomy was performed $(97.9 \%$ in the DCB cases and $44.7 \%$ of BA cases). Procedural success was similar in both groups. More bailout stenting was noted in the balloon angioplasty group versus the DCB group (12.3\% vs $2.13 \%, p=0.044)$ and could reflect a higher use of vessel prepping in the DCB cohort. Freedom from TLR, freedom from limb loss and freedom from major adverse limb events at 2-year follow-up were similar.

In a retrospective study by Stavroulakis et al, ${ }^{17} 47$ consecutive patients with CFA disease were treated using either DCB $(n=26)$ or directional atherectomy with DCB $(n=21)$. Technical success was similar between the two groups. The atherectomy and DCB cohort had numerically better 12-month primary patency at $88 \%$ and freedom from TLR at $89 \%$ when compared to DCB alone $(68 \%$ and $75 \%$, respectively), but neither difference was statistically significant. Furthermore, Cioppa et al $^{18}$ reported prospective data on 30 consecutive patients with severely calcified obstructions of the common femoral artery treated with directional atherectomy and DCB. Chronic total occlusions were present in $6(20 \%)$ cases. Patency and TLR rates at 1 year were $93.4 \%$ and $3.3 \%$, respectively, with a secondary patency rate of $96.7 \%$.

\section{Summary}

Endarterectomy of CFA lesions carries favorable long-term results and is currently considered by many to be the gold standard for treating these lesions. The long-term data for an endovascular approach are lacking and should be balanced against the high initial risk of endarterectomy. Also, it should be emphasized that CFA disease is heterogeneous and infrequently is limited to the CFA only. This adds another complexity to the CFA endarterectomy approach and may have a significant impact on the procedural complication rates and the surgical approach to treatment (hybrid vs not). We believe that the approach to CFA treatment, surgery versus endovascular, is not straightforward and should be a shared decision between the operator and the patient with pros and cons explained in details before an informed consent is obtained. The Inter-Society Consensus for the Management of Peripheral Arterial Disease (TASC II) ${ }^{19}$ favors surgery for type D and low-risk type $\mathrm{C}$ aortoiliac lesions. Type A, B and high-surgical risk type $\mathrm{C}$ lesions are preferably treated with an endovascular approach. CFA lesions are typically grouped in the TASC C and D categories of aortoiliac diseases or 
femoropopliteal diseases. A unilateral external iliac artery stenosis or occlusion (calcified or not) extending into the origin of the CFA is considered TASC C. A chronic total occlusion of the CFA is a TASC D lesion.

Well-controlled trials comparing contemporary endarterectomy to endovascular therapy that incorporate newer therapies such as lithoplasty, atherectomy and drug-coated balloons are needed. Currently, the Percutaneous Intervention versus Surgery in the Treatment of Common Femoral Artery Lesions (PESTO-AFC; www.clinicaltrials.org NCT02517827) is a randomized controlled trial comparing directional atherectomy and DCB to endarterectomy in treating CFA lesions with a planned follow-up for 2 years. It is anticipated that 306 subjects will be enrolled.

\section{Disclosure}

Dr Shammas receives educational and research grants from Bard, Boston Scientific, Intact Vascular, VentureMed Group and Angiodynamics. Dr Shammas also reports involvement for a clinical trial for Shockwave Medical. The authors report no other conflicts of interest in this work.

\section{References}

1. Ballotta E, Gruppo M, Mazzalai F, Da Giau G. Common femoral artery endarterectomy for occlusive disease: an 8-year single-center prospective study. Surgery. 2010;147:268-274. doi:10.1016/j.surg.20 09.08.004

2. Dufranc J, Palcau L, Heyndrickx M, et al. Technique and results of femoral bifurcation endarterectomy by eversion. J Vasc Surg. 2015; 61:728-733. doi:10.1016/j.jvs.2014.09.025

3. Nguyen BN, Amdur RL, Abugideiri M, et al. Postoperative complications after common femoral endarterectomy. J Vasc Surg. 2015;61: 1489-1494. doi:10.1016/j.jvs.2015.01.024

4. Wieker CM, Schönefeld E, Osada N, et al. Results of common femoral artery thromboendarterectomy evaluation of a traditional surgical management in the endovascular era. J Vasc Surg. 2016;64:995-1001. doi:10.1016/j.jvs.2016.04.036

5. Bonvini RF, Rastan A, Sixt S, et al. Endovascular treatment of common femoral artery disease: medium-term outcomes of 360 consecutive procedures. J Am Coll Cardiol. 2011;58:792-798. doi:10.1016/j. jacc.2011.01.070

6. Thiney PO, Millon A, Boudjelit T, Della Schiava N, Feugier P, Lermusiaux P. Angioplasty of the common femoral artery and its bifurcation. Ann Vasc Surg. 2015;29:960-967. doi:10.1016/j.avsg.20 15.02 .001
7. Azéma L, Davaine JM, Guyomarch B, et al. Endovascular repair of common femoral artery and concomitant arterial lesions. Eur J Vasc Endovasc Surg. 2011;41:787-793. doi:10.1016/j.ejvs.2011.02.025

8. Nasr B, Kaladji A, Vent PA, et al. Long- term outcomes of common femoral artery stenting. Ann Vasc Surg. 2017;40:10-18. doi:10.1016/ j.avsg.2016.07.088

9. Siracuse JJ, Van Orden K, Kalish JA, et al.; Vascular Quality Initiative. Endovascular treatment of the common femoral artery in the Vascular Quality Initiative. J Vasc Surg. 2017;65:1039-1046. doi:10.1016/j.jvs.2016.10.078

10. Linni K, Ugurluoglu A, Hitzl W, Aspalter M, Hölzenbein T. Bioabsorbable stent implantation vs. common femoral artery endarterectomy: early results of a randomized trial. $J$ Endovasc Ther. 2014;21:493-502. doi:10.1583/14-4699R.1

11. Goueffic Y, Della Schiava N, Thaveau F, et al. Stenting or surgery for de novo common femoral artery stenosis. JACC Cardiovasc Interv. 2017;10:1344-1354. doi:10.1016/j.jcin.2017.03.046

12. Changal KH, Syed MA, Dar T, Mangi MA, Sheikh MA. Systematic review and proportional meta-analysis of endarterectomy and endovascular therapy with routine or selective stenting for common femoral artery atherosclerotic disease. J Interv Cardiol. 2019;20 19:1593401. doi:10.1155/2019/1593401

13. Soga Y, Tomoi Y, Sato K, Iida O, Yokoi H. Clinical outcome after endovascular treatment for isolated common femoral and popliteal artery disease. Cardiovasc Interv Ther. 2013;28:250-257. doi:10.10 07/s12928-013-0164-1

14. Dattilo PB, Tsai TT, Kevin Rogers R, Casserly IP. Acute and medium term outcomes of endovascular therapy of obstructive disease of diverse etiology of the common femoral artery. Catheter Cardiovasc Interv. 2013;81:1013-1022. doi:10.1002/ccd.v81.6

15. Guo J, Guo L, Tong Z, Gao X, Wang Z, Gu Y. Directional atherectomy is associated with better long term efficiency compared to angioplasty for common femoral artery occlusive disease in Rutherford 2 to 4 patients. Ann Vasc Surg. 2018;51:65-71. doi:10. 1016/j.avsg.2017.12.004

16. Cantu D, Jawaid O, Kokkinidis D, et al. Outcomes of drug-coated balloon angioplasty vs conventional balloon angioplasty for endovascular treatment of common femoral artery atherosclerotic disease. Cardiovasc Revasc Med. 2019;S1553-S8389(19):30751. doi:10.101 6/j.carrev.2019.11.008.

17. Stavroulakis K, Schwindt A, Torsello G, et al. Directional atherectomy with antirestenotic therapy vs drug-coated balloon angioplasty alone for common femoral artery atherosclerotic disease. $J$ Endovasc Ther. 2017;25:92-99. doi:10.1177/1526602817748319

18. Cioppa A, Stabile E, Salemme L, et al. Combined use of directional atherectomy and drug-coated balloon for the endovascular treatment of common femoral artery disease: immediate and one-year outcomes. EuroIntervention. 2017;12:1789-1794. doi:10.4244/EIJD-15-00187

19. Norgren L, Hiatt WR, Dormandy JA, Nehler MR, Harris KA, Fowkes FG, TASC II Working Group. Inter-society consensus for the management of peripheral arterial disease (TASC II). J Vasc Surg. 2007;45(Suppl S):S5-S67. doi:10.1016/j.jvs.2006.12.037
Vascular Health and Risk Management

\section{Publish your work in this journal}

Vascular Health and Risk Management is an international, peerreviewed journal of therapeutics and risk management, focusing on concise rapid reporting of clinical studies on the processes involved in the maintenance of vascular health; the monitoring, prevention and treatment of vascular disease and its sequelae; and the involvement of metabolic disorders, particularly diabetes. This journal is indexed on PubMed Central and MedLine. The manuscript management system is completely online and includes a very quick and fair peerreview system, which is all easy to use. Visit http://www.dovepress. com/testimonials.php to read real quotes from published authors. 